\title{
SPARC, a matricellular protein that functions in cellular differentiation and tissue response to injury
}

\author{
Amy D. Bradshaw and E. Helene Sage \\ Department of Vascular Biology, The Hope Heart Institute, Seattle, Washington, USA \\ Address correspondence to: E. Helene Sage, The Hope Heart Institute, 1124 Columbia Street, Suite 720, Seattle, Washington 98104, USA. \\ Phone: (206) 903-2026; Fax: (206) 903-2044; E-mail: hsage@hopeheart.org.
}

Expressed during many stages of development in a variety of organisms, the matricellular protein SPARC (secreted protein acidic and rich in cysteine, also known as osteonectin or BM-40) is restricted in adult vertebrates primarily to tissues that undergo consistent turnover or to sites of injury and disease (1). The capacity of SPARC to bind to several resident proteins of the ECM, to modulate growth factor efficacy, to affect the expression of matrix metalloproteinases, and to alter cell shape as a counteradhesive factor, supports the idea that SPARC acts to regulate cell interaction with the extracellular milieu during development and in response to injury (Figure 1; see also ref. 1). SPARC is a member of a gene family whose members share structural similarities in one or more protein domains (1). In addition to the numerous studies in cultured cells, the function of SPARC in vivo has been examined primarily in three evolutionarily diverse organisms - Caenorhabditis elegans, Xenopus laevis, and mice. These systems have been used to study the effects of increased or inappropriate SPARC expression, as well as diminished activity resulting from the inactivation of SPARC mRNA, the blocking of protein activity, or mutation of the SPARC gene (Table 1). This Perspective will integrate results from studies in vitro with findings in vivo in an attempt to clarify the current information and to propose functions for SPARC in living tissues.

\section{SPARC in ECM organization}

Vertebrate SPARC binds to a number of different ECM components including thrombospondin 1 , vitronectin, entactin/nidogen, fibrillar collagens (types I, II, III, and $\mathrm{V}$ ), and collagen type IV, the prevalent collagen in basement membranes (1). Therefore, SPARC has the potential to contribute to the organization of matrix in connective tissue as well as basement membranes. Interestingly, SPARC is expressed abundantly in basement membranes and in capsules that surround a variety of organs and tissues. In this regard, SPARC-null mice display early cataractogenesis, a phenotype with $100 \%$ penetrance (2). Transmission electron microscopy of lens epithelial cells in SPARC-null mice shows an intrusion of cellular processes into the basement membrane of the lens capsule, whereas wild-type lens epithelial cells exhibit a precise border at the cell-matrix interface (3). We have proposed that this phenotype reflects aberrant cell behavior or differentiation resulting from altered composition or structure of the basement membrane formed in the absence of SPARC.

\section{Figure 1}

Structure of SPARC protein. A ribbon diagram derived from crystallographic data shows the three modular domains of SPARC. Representative activities attributed to each domain are shown beneath the designated amino acids. The follistatin-like domain contains the peptide 2.1 (see ref. 1) shown in green and the (K)GHK angiogenic peptide (amino acids 114-130) shown in black. The E-C domain contains the amino acids 255-274 (peptide 4.2) shown in yellow. Modified from Hohenester et al. (29) and the Brookhaven Protein Database, accession no. $1 \mathrm{BM} 0$ from Ref. 1, with permission.
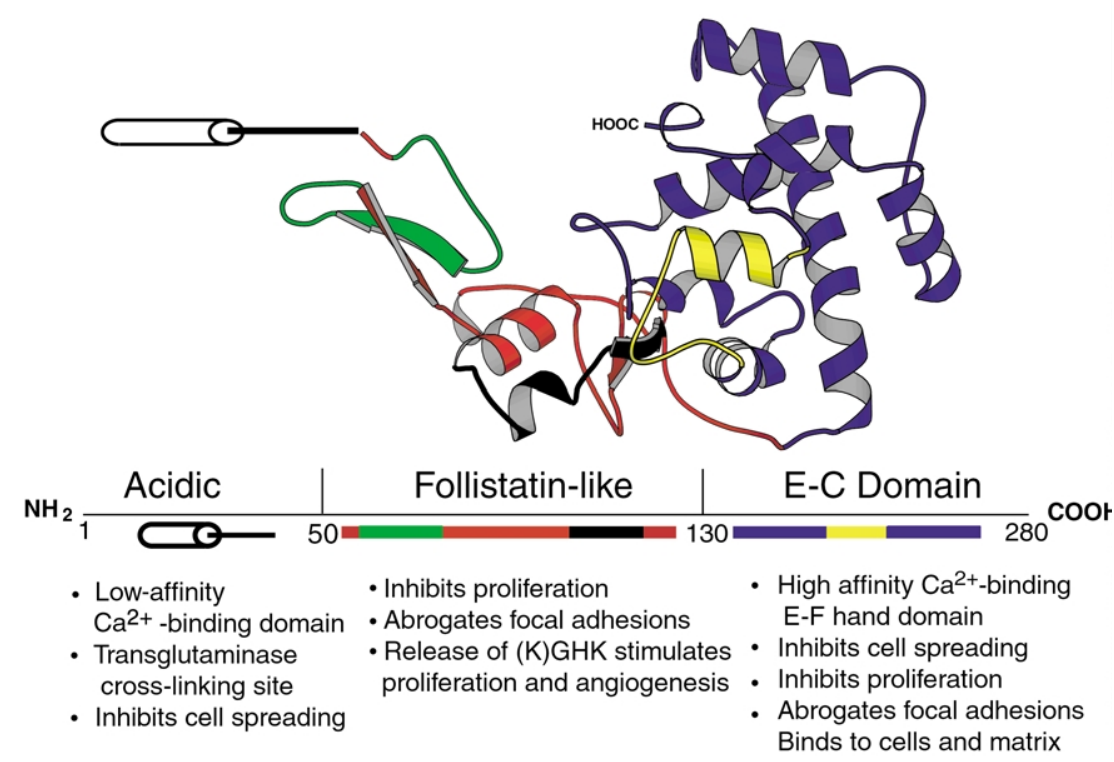


\section{Matricellular proteins

Table 1

Phenotypic abnormalities resulting from inactivation of SPARC in vivo

\begin{tabular}{|c|c|c|c|}
\hline Organism & Technique & Phenotype & Reference \\
\hline \multirow[t]{3}{*}{ C. elegans } & cRNA interference & Embryonic lethality & 9 \\
\hline & & Lack of gut granules & \\
\hline & & Nonfunctional gonads & \\
\hline \multirow[t]{2}{*}{$X$. laevis } & Antibody block & Bent and shortened embryonic axes & 28 \\
\hline & & Abnormal eye development & \\
\hline \multirow[t]{6}{*}{ Mus musculus } & Transgenic-null & Cataractogenesis & 2 (and references therein) \\
\hline & & Accelerated dermal wound healing & A.D. Bradshaw et al., unpublished \\
\hline & & Aberrant dermal collagen fibrils & A.D. Bradshaw et al., unpublished \\
\hline & & Curly tails & \\
\hline & & Osteopenia & 17 \\
\hline & & Increased fat deposition & $\begin{array}{c}\text { A.D. Bradshaw and E.H. Sage, } \\
\text { unpublished }\end{array}$ \\
\hline
\end{tabular}

With respect to connective tissue, preliminary transmission electron microscopy of dermal collagen fibers also revealed differences between wild-type and SPARCnull mice. Whereas collagen fibrils from wild-type skin exhibit a variety of large and small diameters, as observed previously in normal adult animals, SPARCnull fibrils are smaller and more uniform in diameter (A.D. Bradshaw et al., unpublished results). Differences in collagen fibril size are consistent with our primary observation that the skin of SPARC-null mice is more easily stretched and weaker in tensile strength than that of wild-type mice. Apparently the absence of SPARC affects collagen fibrillogenesis, most likely during development, although confirmation of this idea awaits completion of experiments in which a developmental time course of collagen fibril assembly will be analyzed. Whether SPARC acts to affect fibrillogenesis directly through its collagen-binding capacity or by another mechanism is unknown. However, in connective tissues of mov-13 mice, which are deficient in collagen I, SPARC is not distributed in specific matrices that are known sites of SPARC deposition in wild-type embryos (1). In developing Xenopus, SPARC appears to be concentrated within the intersomitic furrows, a location known to be rich in collagen fibers (4).

Further evidence for the importance of SPARC in connective tissue is found in the curly tails of SPARC-null mice, a characteristic reminiscent of thrombospondin 2 -null mice (5). Thrombospondin 2 is another matricellular protein with potential collagen-binding activity, as thrombospondin 2-null mice also display aberrant collagen fibrils in the skin and an abnormally flexible tail (5). In fact, a variety of ECM-associated components have been implicated in collagen fibrillogenesis by virtue of the phenotypic abnormalities observed in transgenic mice with targeted deletions of the genes for decorin, fibromodulin, lumican, and osteopontin, among others (6-8). Since some of these proteins and proteoglycans are known to affect collagen fibrillogenesis in vitro, phe- notypic abnormalities in collagen fibrils were not unexpected in these animals. Others, such as SPARC and thrombospondin 2, proved to be more surprising. Clearly the assembly and regulation of collagen fibril size is a complex process about which a great deal remains to be learned.

Similar to vertebrate SPARC, C. elegans SPARC is encoded by a single gene, ost -1 . Although there are both structural and functional differences between vertebrate and nematode SPARC, such as a reduced affinity for $\mathrm{Ca}^{2+}$, binding to both collagen types I and IV is conserved (1). SPARC in C. elegans is expressed primarily by body wall and sex muscle cells, although SPARC protein is also associated with the basement membrane of the pharynx, a tissue in which SPARC mRNA is not detected (9). A similar disparity between sites of synthesis and deposition has been noted in C. elegans for collagen IV, and in mouse, for SPARC (1). Thus, nematode SPARC appears to be transported extracellularly to basement membranes at some distance from its sites of synthesis, an observation suggesting a function for this protein in matrix organization or activity.

The capacity of SPARC to bind to a number of different ECM proteins provides a basis for the association of SPARC with both basement membranes and fibrous connective tissue. During development, when many ECMs are being laid down, higher levels of SPARC are observed (1). Subsequently, the expression of SPARC is restricted to sites of ECM turnover and is virtually undetectable in normal cells within their established ECM. In fact, Damjanovski et al. observed in Xenopus embryos that expression of SPARC decreased precipitously upon morphological differentiation of specific tissues (10).

Interestingly, SPARC is a substrate of transglutaminase, an enzyme that establishes covalent cross-links between proteins (11). Although the function of tissue transglutaminase in the stabilization of the ECM is not completely understood, strong evidence for its importance in matrix assembly and cell interaction with ECM is emerging (12). Possibly, expression of SPARC in response to injury or tissue remodeling is necessary to facilitate production of an ECM permissive for cell migration, proliferation, and differentiation. SPARC might substitute for other transglutaminase substrates that provide structural support in the ECM: for example, cross-linking of SPARC instead of fibronectin, another substrate of transglutaminase, could result in a more malleable matrix. 


\section{SPARC and growth factors}

SPARC binds to and decreases the mitogenic potency of both PDGF and VEGF, in part by its abrogation of growth factor-receptor interaction $(1,2)$. In addition, SPARC can counteract the proliferative capacity of bFGF on smooth muscle cells, although physical interaction of SPARC with bFGF remains to be determined $(1,2)$. Furthermore, addition of SPARC to myoblasts promotes differentiation through inhibition of bFGFinduced proliferation (K. Motamed et al., unpublished results). That SPARC binds ECM components and modulates the growth factor-induced proliferation (and, in at least one case, differentiation) of at least three separate mitogens adds another dimension to the function of SPARC in the regulation of cell behavior.

In addition to PDGF, VEGF, and bFGF, SPARC can also modulate the activity of TGF- $\beta$, as seen in recent studies with SPARC-null mesangial cells. In culture, these cells express decreased levels of TGF- $\beta$ mRNA and protein in comparison with wild-type mesangial cells (1). Addition of recombinant SPARC to either wild-type or SPARC-null cells increases TGF- $\beta$ mRNA and protein and is associated with an increase in collagen I production. Interestingly, TGF- $\beta$ can interact with the ECM through the binding of latent TGF- $\beta$ binding protein (LTBP-1), which is cross-linked to the ECM in a transglutaminase-dependent manner and facilitates activation of latent TGF- $\beta$ in endothelial cells (13). The lack of SPARC might lead to aberrant assembly of ECM that in turn affects the localization and subsequent activation of TGF- $\beta$.

\section{SPARC as a counteradhesive protein}

The capacity for SPARC to modulate growth factor activity has far-reaching implications for many aspects of cell behavior that include proliferation, migration, and differentiation. Perhaps equally important, however, is the counteradhesive activity associated with SPARC. For over a decade, SPARC has been appreciated as a modifier of cell shape (1). Addition of purified SPARC to many cell types in culture induces cell rounding, a process thought to be distinct from the inhibition of cell cycle by SPARC. Cell rounding activity is sensitive to tyrosine kinase inhibitors, whereas inhibition of proliferation depends in part upon a pertussis toxin-sensitive pathway, at least in vascular smooth muscle cells (1). Focal adhesions dissociate upon addition of purified SPARC to endothelial cell cultures, although the mechanism by which SPARC influences cell shape is not completely understood (14). Whether SPARC acts upon cell surfaces through a specific receptor or acts by blocking adhesive interactions is unclear. Indeed these possibilities might each apply in different situations and would thereby allow cells of various types to respond to SPARC in a characteristic manner (see Murphy-Ullrich, this Perspective series, ref. 15).

Cell shape, like growth factor activity, can influence cell proliferation, migration, and differentiation. The evidence that SPARC acts to modulate such processes in vivo is becoming substantially more credible, based on experiments performed in C. elegans, Xenopus, and transgenic mice that do not express SPARC. The counteradhesive function of SPARC appears to be conserved in the diverse organisms that express the protein. Thus, overexpression of SPARC in C. elegans leads to an uncoordinated phenotype that probably reflects the compromised ability of the body wall muscle cells to attach to the adjacent basement membrane and to generate force (1). Huynh et al. (16) have tested the effects on vertebrate development of a peptide representing the $\mathrm{COOH}$-terminal $\mathrm{Ca}^{2+}$-binding domain of SPARC (Figure 1, E-C domain). This peptide mimics the full-length protein in its ability to provoke changes in cell shape (1). When injected into developing Xenopus embryos, it caused the rounding of migrating mesodermal cells and severe developmental abnormalities - incomplete gastrulation and a reduction in anterior structures (16). The severe cataracts seen at early ages in SPARC-null mice might also arise because of abnormalities in cell shape. As shown in Figure 2a, differentiating lens epithelial cells (shown in cross sections from the cortex of the lens from 3-month-old animals) normally exhibit an ordered structure of uniformly shaped cells. Agematched SPARC-null lens cells (Figure $2 b$ ) display irregular shapes and alignment. Whether the changes in cell shape that occur during the normal development of the lens are due to the direct action of SPARC or to a secondary effect occurring later in the differentiation process is currently unknown.

\section{Figure 2}

SPARC-null lens epithelial cells display changes in cell shape and alignment in comparison with wild-type cells. Sections from similar areas of wild-type and SPARC-null lens were stained with hematoxylin and eosin. Cortical fibers from a 3month-old wild-type lens (a) are regular and precisely aligned, whereas those from a SPARC-null lens (b) are irregular in shape and alignment. Magnification, $\times 220$.
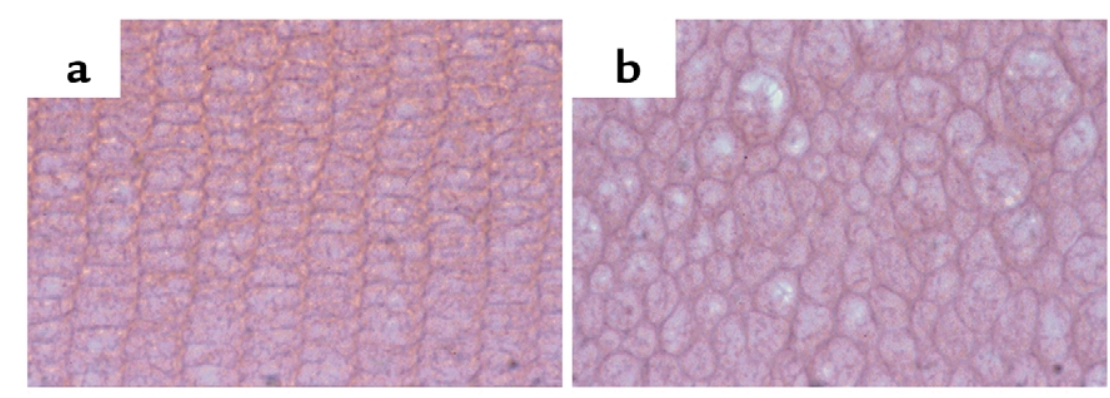


\section{Matricellular proteins}

E. Helene Sage, Series Editor
A recent study of proteins that influence morphineinduced locomotor sensitivity in the amygdala identified SPARC as important in this process. It is believed that prolonged exposure to morphine leads to physiological changes in the brain that might be dependent upon synaptic rearrangement. Ikemoto et al. found expression of SPARC in the amygdala, the region of the brain known to control locomotor sensitivity, in rats exposed to morphine (17). In fact, administration of recombinant SPARC directly to the amygdala resulted in locomotor sensitivity in the absence of morphine. The authors also showed that SPARC induces shape changes in neurons, as previously reported for several other cell types (1). Perhaps the counteradhesive activity of SPARC facilitates neuronal rearrangement in vivo, including synaptic plasticity, with its attendant physiological and behavioral implications.

\section{SPARC and differentiation}

The body wall muscle dysfunction seen in nematodes with aberrant expression of SPARC has been pursued by cRNA interference to block the production of SPARC in these organisms. The resulting phenotypes ranged from embryonic lethality to surviving adults with significant morphological abnormalities, including smaller adults, a lack of gut granules, and no functional gonads (Table 1; ref. 9). Because the major site of SPARC mRNA transcription in C. elegans occurs in the body wall muscle cells, embryonic death has been attributed to defects in muscle function, as no obvious differences in morphology are evident at this stage. Smaller adults might result from a nutritional defect caused by the lack of gut granules. Whether the absence of SPARC affects the ECM upon which precursor cells migrate and receive appropriate signals for differentiation into gut granules, or whether another developmental mechanism is disturbed, remains to be determined. Clearly, functional interruption of SPARC activity in both C. elegans and Xenopus (Table 1) has severe consequences that most certainly involve differentiation, although the molecular mechanisms are currently undefined.

Lens epithelial cells differentiate at specific points along the lens periphery, during which cells lose contact with the capsular basement membrane, elongate anteriorly and posteriorly, lose their organelles, and eventually form the highly ordered, transparent structure of the lens. As SPARC is a component of the lens capsule, the lack of SPARC might result in a significantly altered ECM with respect to structure and/or growth factor deposition (2). A hitherto unidentified function of SPARC might also be critical for these highly specialized cells, such as the regulation of intercellular adhesive proteins, e.g., certain of the cadherins, and gap junction proteins, which are critical for the differentiation of lens fibers.

In vertebrates, SPARC is a major noncollagenous component of bone. Delaney et al. recently reported a severe bone phenotype associated with SPARC-null mice that was manifested as adult osteopenia (18). The absence of SPARC led to substantial decreases in bone mass, the severity of which increased with age. Interestingly, we have observed significant increases in subcutaneous fat in SPARC-null relative to wild-type mice, a difference that also appears to increase with age. Other fat deposits in SPARC-null mice are increased in size as well, in comparison with those of wild-type animals, although overall body weight is not substantially different. Both osteoblasts and adipocytes originate from common progenitor cells present in the bone marrow (19). The decrease in bone mass and the increase in fat deposition might reflect differentiation events that favor the formation of adipocytes over that of osteoblasts. Indeed, SPARC might act directly to influence cell fate in this example; conversely, the absence of SPARC might affect ECM assembly and/or localization of growth factors that in turn could influence differentiation. Rodriguez et al. recently reported an analysis of the differentiative capacity of mesenchymal stem cells isolated from postmenopausal women with and without osteoporosis (20). Cells from osteoporotic patients produced less TGF- $\beta$ and collagen I and exhibited an increased incidence of adipogenic differentiation in culture. Seemingly, the deficiencies in TGF- $\beta$ and hence the capacity to synthesize a collagen I-rich ECM favored adipose formation over bone formation. Since a decrease in TGF- $\beta$ and collagen I is observed in SPARC-null mesangial cells versus wild-type cells, a similar mechanism might be in place to influence mesenchymal stem cell differentiation as well.

That SPARC-null mice develop to adulthood and reproduce with a grossly normal skeleton and fat deposits argues against a direct influence of SPARC in decisions regarding cell fate. A more plausible hypothesis is that subtle differences in ECM configuration and/or growth factor activity in animals lacking SPARC lead eventually to tissue failure, especially when the animal is challenged with injury or disease.

\section{SPARC and wound healing}

Dermal wound healing involves many processes that could be affected by the absence of SPARC: ECM turnover and reassembly, cell migration, cell proliferation, and recruitment/differentiation of connective tissue cells. Perhaps surprisingly, we have found that excisional dermal wounds produced in SPARC-null mice close more rapidly, relative to those in wild-type mice (A.D. Bradshaw et al., unpublished results). The mechanisms by which SPARC could influence dermal closure and/or healing are many. One possibility is the capacity of SPARC to influence the activity of TGF- $\beta$. Recently, Ashcroft et al. reported that mice deficient in Smad-3 (a downstream signaling molecule in the TGF$\beta$ pathway) also display accelerated dermal wound healing, in comparison with wild-type mice (21). Although TGF- $\beta$ has been shown to augment healing when administered topically, the signaling pathways in vivo 
are complex. Local and probably cell-specific inhibition of TGF- $\beta$ signaling apparently enhances dermal healing. Thus, the absence of SPARC might decrease the levels of active TGF- $\beta$, and consequently its availability to certain cells, during dermal healing and would thereby accelerate the process. In addition, the capacity of SPARC to inhibit PDGF, bFGF, and VEGF, three factors that have been shown to improve healing, might also contribute to the enhancement of wound closure in the absence of SPARC.

The structure of the dermis in SPARC-null mice, in particular the collagen fibrils, is clearly different from that in wild-type mice. The decrease in size of the collagen fibrils, perhaps with other alterations in the ECM, might result in a more permissive milieu for cellular infiltration and healing. It is likely that the SPARC-null ECM would be more easily degraded by matrix metalloproteinases. In addition, overall wound contraction might be more easily achieved with a SPARC-null matrix, given that it appears to be less structured and hence possibly easier to contract than that of wild-type mice.

Another contribution to enhanced wound closure might be the increase in subdermal fat that we have observed in SPARC-null mice. Many of the cells that invade the wound bed to repair the injured tissue have been shown to originate in the subdermal fat and muscle layers (22). Perhaps an increase in the amount of fat enhances the availability of cells that contribute to wound closure and thus accelerates the healing process. Moreover, Frank et al. reported recently that leptin, a factor produced by adipose tissue, enhances dermal wound healing in mice (23). An increase in the subcutaneous fat layer in SPARC-null mice most likely results in increased levels of leptin in the skin. Thus accelerated closure might reflect an increase in leptin levels at the wound site.

\section{Cancer and angiogenesis}

Similar to wound healing, substantial ECM turnover and rearrangement also occur during tissue invasion by tumor cells. High levels of SPARC are often associated with metastatic tumors (1). In fact, SPARC has been proposed as a diagnostic marker of invasive meningiomas (24). Inhibition of SPARC expression by antisense RNA diminished both adhesive and invasive capacities of human melanoma cells in vitro and in vivo. Moreover, melanoma cells with suppressed expression of SPARC were no longer able to generate tumors when injected into athymic mice, whereas control melanoma cells showed $100 \%$ tumorigenicity (25).

Angiogenesis, the growth of new vessels from extant vasculature, is a major factor in tumor growth and metastasis (26). Since neovascularization includes endothelial cell invasion and ECM remodeling, it was not surprising to find that SPARC is expressed by endothelial cells in culture and in tissues (1). Notably,
SPARC has been shown to be associated with growing vessels in the chick chorioallantoic membrane, a highly vascular structure that provides nutrients and gas exchange for the growing embryo (1). Interestingly, the authors reported the generation of proteolytic fragments from SPARC that were localized to the tips of growing vessels. The $\mathrm{Cu}^{2+}$-binding peptide $\mathrm{GHK}$, previously characterized as an angiogenic compound in plasma, as well as the more potent stimulator KGHK, could be derived from SPARC (Figure 1; see also ref. 1). To date, SPARC is the only extracellular protein found to contain the sequence KGHK, which is conserved throughout vertebrate SPARC (1). The existence of regions in SPARC that can be released by proteolysis and that display activities different from those of the native, intact protein somewhat complicates our understanding of the functions of SPARC, but at the same time offers new possibilities for modulation of cell-matrix interactions (27).

We have recently performed a sponge invasion assay in SPARC-null mice to determine whether angiogenesis in the dermis is abnormal. Animals lacking SPARC displayed a substantial increase in the amount of fibrovascular invasion of subcutaneous polyvinyl alcohol sponges in comparison with that of wild-type mice (A.D. Bradshaw et al., unpublished results). Whether an increase in invasion and angiogenesis reflects a more permissive milieu for cell migration and proliferation, due to an altered ECM, or an increase in the availability of angiogenic growth factors such as PDGF and VEGF, remains to be determined. Also of interest is the use of SPARC-null mice in selected tumor models to determine whether metastatic potential or tumor angiogenesis is affected by the absence of SPARC.

\section{Concluding remarks and future directions}

That SPARC is expressed by organisms as diverse as $C$. elegans and humans is testimony to the fundamental importance of this protein to multicellular life. Since invertebrate SPARC does not bind hydroxyapatite, the SPARC gene has apparently become specialized for an additional function unique to vertebrates, i.e., bone formation. The existence of other SPARC family members, such as SC1, provides another layer of complexity with regard to compensatory activities $(1,2)$. SPARC might influence cell behavior through its interactions with cell surfaces, growth factors, and ECM. Elucidating the mechanisms by which SPARC influences cell migration, proliferation, and differentiation will contribute to our understanding of a variety of different biological processes including development, wound healing, angiogenesis, and cancer. Although significant progress has been made in the characterization of SPARC, a substantial amount of information remains to be unearthed, with results which undoubtedly will prove to be as provocative as they are gratifying. 


\section{Acknowledgments}

We would like to thank the members of the Sage laboratory for helpful discussions and ideas. Qi Yan contributed the photograph in Figure 2. This work was supported by NIH grants GM-40711 and HL-59475 to E.H. Sage, and DK-07467 to A.D. Bradshaw.

1. Brekken, R.A., and Sage, E.H. 2000. SPARC, a matricellular protein: at the crossroads of cell-matrix communication. Matrix Biol. 19:569-580.

2. Yan, Q., and Sage, E.H. 1999. SPARC, a matricellular glycoprotein with important biological functions. J. Histochem. Cytochem. 47:1495-1505.

3. Norose, K., Lo, W.-K., Clark, J.I., Sage, E.H., and Howe, C.C. 2000. Lenses of SPARC-null mice exhibit an abnormal cell surface-basement membrane interface. Exp. Eye Res. 71:295-307.

4. Ringuette, M., Drysdale, T., and Liu, F. 1992. Expression and distribution of SPARC in early Xenopus laevis embryos. Roux's Archives of Developmental Biology. 202:4-9.

5. Kyriakides, T.R., et al. 1998. Mice that lack thrombospondin 2 display connective tissue abnormalities that are associated with disordered collagen fibrillogenesis, an increased vascular density, and a bleeding diathesis. J. Cell Biol. 140:419-430.

6. Danielson, K.G., et al. 1997. Targeted disruption of decorin leads to abnormal collagen fibril morphology and skin fragility. J. Cell Biol. 136:729-743.

7. Ezura, Y., Chakravarti, S., Oldberg, A., Cheroneva, I., and Birk, D.E. 2000 Differential expression of lumican and fibromodulin regulate collagen fibrillogenesis in developing mouse tendons. J. Cell Biol. 151:779-788.

8. Liaw, L., et al. 1998. Altered wound healing in mice lacking a functional osteopontin gene (spp1). J. Clin. Invest. 101:1468-1478.

9. Fitzgerald, M.C., and Schwarzbauer, J.E. 1998. Importance of the basement membrane protein SPARC for viability and fertility in Caenorbabditis elegans. Curr. Biol. 8:1285-1288.

10. Damjanovski, S., Malaval, L., and Ringuette, M.J. 1994. Transient expression of SPARC in the dorsal axis of early Xenopus embryos: correlation with calcium-dependent adhesion and electrical coupling. Int. J. Dev. Biol. 38:439-446.

11. Aeschlimann, D., Kaupp, O., and Paulsson M. 1995. Transglutaminasecatalyzed matrix cross-linking in differentiating cartilage: identification of osteonectin as a major glutaminyl substrate. J. Cell Biol. 129:881-892.

12. Greenberg, C.S., Birchbichler, P.J., and Rice, R.H. 1991. Transglutaminases: multifunctional cross-linking enzymes that stabilize tissues. FASEB J. 5:3071-3077.

13. Nunes, I., Gliezes, P.-M., Metz, C.N., and Rifkin, D.B. 1997. Latent transforming growth factor- $\beta$ binding protein domains involved in activation and transglutaminase-dependent cross-linking of latent transforming growth factor- $\beta$. J. Cell Biol. 136:1151-1163.
14. Murphy-Ullrich, J.E., Lane, T.F., Pallero, M.A., and Sage, E.H. 1995 SPARC mediates focal adhesion disassembly in endothelial cells through a follistatin-like region and the calcium-binding EF-hand.J. Cell. Biochem. 57:341-350.

15. Murphy-Ullrich, J.E. 2001. The de-adhesive activity of matricellular proteins: is intermediate cell adhesion an adaptive state? J. Clin. Invest. 107:785-790.

16. Huynh, M.-H., Sage, E.H., and Ringuette, M. 1999. A calcium-binding motif in SPARC/osteonectin inhibits chordomesoderm cell migration during Xenopus laevis gastrulation: evidence of counter-adhesive activity in vivo. Dev. Growth Differ. 41:407-418.

17. Ikemoto, M., Takita, M., Imamura, T., and Inoue, K. 2000. Increased sensitivity to the stimulant effects of morphine conferred by anti-adhesive glycoprotein SPARC in amygdala. Nat. Med. 6:910-915.

18. Delaney, A.M., et al. 2000. Osteopenia and decreased bone formation in osteonectin-deficient mice. J. Clin. Invest. 105:915-923.

19. Haynesworth, S.E., Goshima, J., Goldberg, V.M., and Caplan, A.I. 1992 Characterization of cells with osteogenic potential from human marrow. Bone. 13:81-88.

20. Rodriguez, J.P., Montecinos, L., Rios, S., Reyes, P., and Martinez, J. 2000. Mesenchymal stem cells from osteoporotic patients produce a type I collagen-deficient extracellular matrix favoring adipogenic differentiation. J. Cell. Biochem. 79:557-565.

21. Ashcroft, G.S., et al. 1999. Mice lacking smad-3 show accelerated wound healing and an impaired local inflammatory response. Nat. Cell Biol. 1:260-266.

22. Kurman, M., and Argyris, T.S. 1975. The proliferative response of epidermis of hairless mice to full thickness wounds. Am. J. Pathol. 79:301-310.

23. Frank, S., Stallmeyer, B., Kampfer, H., Kolb, N., and Pfeilschifter, J. 2000. Leptin enhances wound re-epithlelialization and constitutes a direct function of leptin in skin repair. J. Clin. Invest. 106:501-509.

24. Rempel, S.A., Ge, S., and Gutierrez, J.A. 1999. SPARC: a potential diagnostic marker of invasive meningiomas. Clin. Cancer Res. 5:237-241.

25. Ledda, M.F., et al. 1997. Suppression of SPARC expression by antisense RNA abrogates the tumorigenicity of human melanoma cells. Nat. Med. 3:171-176.

26. Folkman, J. 1995. Angiogenesis in cancer, vascular, rheumatoid and other disease. Nat. Med. 1:27-31.

27. Sage, E.H. 1997. Pieces of eight: bioactive fragments of extracellular proteins as regulators of angiogenesis. Trends Cell Biol. 7:182-186.

28. Purcell, L., Gruia-Gray, J., Scanga, S., and Ringuette, M. 1993. Developmental anomalies of Xenopus embryos following microinjection of SPARC antibodies. J. Exp. Zool. 265:153-164.

29. Hohenester, E., Maurer, P., and Timpl, R. 1997. Crystal structure of a pair of follistatin-like and EF-hand calcium-binding domains in BM-40. EMBO J. 16:3778-3786.

30. Protein Data Bank. http://www.rcsb.org/pdb/. 